\title{
Subarachnoid Hemorrhage from Ruptured Aneurysm in the Middle Cerebral Artery and Anterior Cerebral Artery Trunk: A Single-Center Study
}

\author{
Woowon Oh', Yeongu Chung ${ }^{1}$, Jebeom Hong ${ }^{1}$, Yu Sam Won ${ }^{1}$, Pil-Wook Chung', Myung Ho Rho ${ }^{3}$ \\ Departments of ${ }^{1}$ Neurosurgery, ${ }^{2}$ Neurology, ${ }^{3}$ Radiology, Kangbuk Samsung Hospital, Sungkyunkwan University School of Medicine, \\ Seoul, Republic of Korea
}

Corresponding author:

Yu Sam Won

Department of Neurosurgery, Kangbuk Samsung Hospital,

Sungkyunkwan University School of Medicine, 29 Saemunan-ro, Jongno-gu, Seoul 03181, Republic of Korea

Tel: $+82-2-2001-2159$

Fax: $+82-2-2001-2157$

E-mail: yusam.won@samsung.com

Received: July 11, 2021

Revised: August 24, 2021

Accepted: August 26, 2021
Objective: Objective: Ruptured anterior cerebral artery (ACA) trunk aneurysms and middle cerebral artery (MCA) trunk aneurysms are rare, and little is known about them. This study was conducted to determine the difference between these and other types of aneurysms.

Methods: We performed a retrospective review of patients diagnosed with subarachnoid hemorrhage over an 8-year period at a single institution. We analyzed the characteristics, clinical factors, and radiological components of aneurysms at the trunk portion of A-1 and M-1. Descriptive analysis and univariate analysis for factors were performed to determine the differences of ACA A-1 portion trunk and MCA M-1 portion trunk aneurysms from other ACA and MCA aneurysms, respectively. Results: Univariate logistic regression modeling showed that trunk aneurysms in MCA M-1 had a smaller dome size $(p=0.026)$ and dome/neck ratio $(p=0.048)$ than other MCA aneurysms. Likewise, through univariate logistic regression modeling, the ACA group showed differences in dome size including age $(p=0.001)$ as well as dome size $(p=0.038)$ and dome neck ratio $(p=0.041)$ in the A1 region. Conclusion: MCA M-1 and ACA A-1 trunk aneurysms are likely to have several different characteristics such as small in size and a lower dome/neck ratio. Also, due to their close locations to the perforator arteries, there is a high possibility of perforator artery injury when treating these aneurysms. Thus, careful attention is required when setting the treatment methods, and further studies about these aneurysms are needed.

Key Words: Anterior cerebral artery; Intracranial aneurysm; Middle cerebral artery; Subarachnoid hemorrhage

\section{INTRODUCTION}

There are many previous and ongoing studies regarding pathologies, risk factors, treatment options, and prognoses of spontaneous subarachnoid hemorrhage (SAH) from ruptured aneurysm due to its severe neurologic deficits and high mortality despite treatment ${ }^{15}$. Further, frequent sites of ruptured aneurysms are middle cerebral artery (MCA) bifurcation (MCAB), anterior communicating artery (AComA), and posterior communicating artery $(\mathrm{PComA})^{16,17)}$. The distribution of cerebral aneurysms among those sites is based on pathophysiology and hemodynamics factors including flow impingements, pressure, and wall shear stress ${ }^{2,19}$.

Rarely are ruptured aneurysms observed at the anterior cerebral artery (ACA) and the MCA trunk. Investigation of pathophysiologichemodynamic factors and anatomy of the cerebral artery suggest that these rare aneurysms originate from the bifurcation of perforator arteries. Because these aneurysms are so rare, there are very few related studies. What is known is that ACA A-1 portion and MCA M-1 portion aneurysms are rare (4\%), small in size, and associated with other variant anatomies such as ACA aplasia ${ }^{1,6,10,11,13,14,18,20)}$.

The study aimed to explore several representative cases of trunk aneurysms and determine the differences between ruptured trunk aneurysms and other aneurysms as well as to define other characteristics of these aneurysms. We also show two cases of these rare ruptured aneurysms to provide additional information.

\section{MATERIALS AND METHODS}

\section{Study Design and Population}

In this study, we retrospectively reviewed 329 patients diagnosed with spontaneous SAH from January 2012, to March 2020, at our institute.

First of all, to understand the basic information of ruptured aneurysmal SAH, various demographics and characteristics of all 321 patients excluding 6 patients who failed to perform transfemoral 
cerebral angiography (TFCA) for various reasons and 2 patients who were angio-negative were reviewed.

Secondly, to evaluate the comparison of ACA A-1 portion aneurysms and other ACA aneurysms and MCA M-1 portion aneurysms and other MCA aneurysms respectively, we also excluded 80 patients with ruptured SAH at internal carotid artery (ICA), 29 with posterior circulation aneurysms, and 1 with the recurrent artery of Heubner. After all, 93 patients with ruptured aneurysms at the MCA and 121 with ruptured aneurysms at the ACA were included. Exclusion of patients was carried out as shown in Fig. 1.

We retrospectively reviewed the electronic medical records (EMR) and radiologic findings for medical history; laboratory findings; neurologic grade; and the distribution, shape, and size of past aneurysms. Blood analysis findings and TFCA records of the patients at admission were reviewed.

\section{Assessment of Trunk Aneurysm}

We defined 'runk aneurysms' as aneurysms originating from MCA M-1 portion or ACA A-1 portion trunk. Specifically, ACA A-1 trunk aneurysms were defined as those located between ICA bifurcation (ICAB) and AComA, while MCA trunk aneurysms were defined as those located between $I C A B$ and $M C A B$. Aneurysms originating from the anterior temporal artery, which is frequently located on the MCA M-1 trunk, were excluded. The distribution, shape, and size of each aneurysm were identified manually on EMR and radiologicresults.

\section{Statistical Analysis}

The influencing factors included age, sex, medical history (hypertension, diabetes mellitus, and ischemic heart disease), laboratory findings (high-density lipoprotein, low-density lipoprotein, total cholesterol, hemoglobin Alc), blood pressure, locations, modified Fisher grade, World Federation of Neurological Surgeons (WFNS) score, and size of aneurysm. The student's t-test was used to evaluate differences in normally distributed continuous variables.

Descriptive analysis for the probable factors in $\mathrm{SAH}$ patients was performed (Table 1).

Univariate analysis of probable factors in MCA and ACA groups

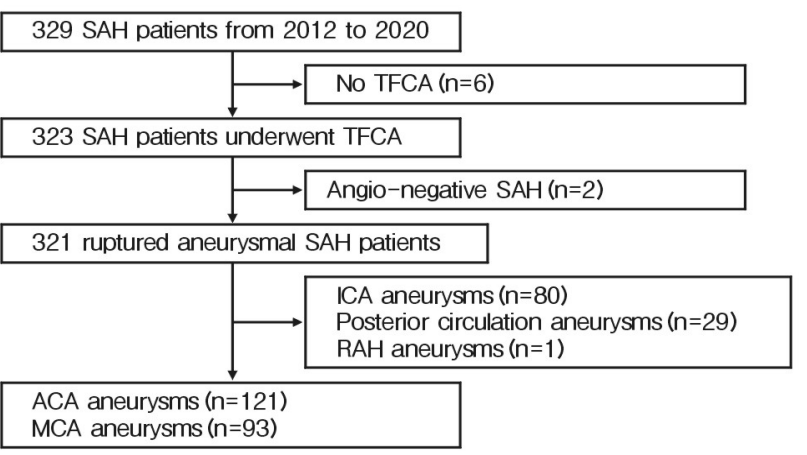

Fig. 1. Exclusion criteria. SAH: subarachnoid hemorrhage; TFCA: transfemoral cerebral angiography. was carried out for comparison of trunk aneurysms (Table 2, 3).

The $\chi^{2}$ test was utilized to evaluate differences in categorical variables. The Mann-Whitney U-test was applied when comparing continuous variable between two groups without normal distribution and when the number of at least one group was less than 10 patients. The Kruskal Wallis test was used to evaluate comparisons in categorical factors among more than two groups. All numerical values were calculated using R soffware version 4.1.0 (The R Foundation

Table 1. Characteristics in SAH patients

\begin{tabular}{|c|c|}
\hline Variables & SAH patients $(n=321)$ \\
\hline Age (years) & $58.53 \pm 13.99$ \\
\hline Sex (female) & 198 (61.7\%) \\
\hline \multicolumn{2}{|l|}{ Medical history } \\
\hline Hypertension & 132 (41.1\%) \\
\hline Diabetes mellitus & 21 (6.5\%) \\
\hline Ischemic heart disease & $17(5.3 \%)$ \\
\hline \multicolumn{2}{|l|}{ Laboratory findings } \\
\hline $\mathrm{HDL}(\mathrm{mg} / \mathrm{dL})$ & $54.80 \pm 14.39$ \\
\hline LDL (mg/dL) & $100.18 \pm 33.44$ \\
\hline Total cholesterol (mg/dL) & $165.89 \pm 64.469$ \\
\hline HbAlc (\%) & $5.66 \pm 0.53$ \\
\hline Systolic BP at ER (mmHg) & $160.60 \pm 33.94$ \\
\hline Diastolic BP at ER (mmHg) & $88.78 \pm 17.42$ \\
\hline \multicolumn{2}{|l|}{ Location } \\
\hline $\mathrm{ACA}$ & 122 (38.0\%) \\
\hline MCA & 90 (28.0\%) \\
\hline ICA & $80(24.9 \%)$ \\
\hline Posterior circulation & $29(9.0 \%)$ \\
\hline \multicolumn{2}{|l|}{ Modified Fisher grade } \\
\hline । & $2(0.6 \%)$ \\
\hline$\|$ & 52 (16.2\%) \\
\hline III & 171 (53.3\%) \\
\hline IV & $96(29.9 \%)$ \\
\hline \multicolumn{2}{|l|}{ WFNS grade } \\
\hline 1 & 142 (44.2\%) \\
\hline$\|$ & 42 (13.1\%) \\
\hline III & $18(5.6 \%)$ \\
\hline IV & $63(19.6 \%)$ \\
\hline V & $56(17.4 \%)$ \\
\hline \multicolumn{2}{|l|}{ Aneurysm shape ( mm) } \\
\hline Dome & $5.24 \pm 3.03$ \\
\hline Neck & $3.42 \pm 1.70$ \\
\hline Dome/Neck & $1.66 \pm 0.93$ \\
\hline
\end{tabular}

SAH: subarachnoid hemorrhage; HDL: high-density lipoprotein; LDL: low-density lipoprotein; HbAlc: glycated hemoglobin; BP: blood pressure; ER: emergency room; ACA: anterior cerebral artery; MCA: middle cerebral artery; ICA: internal carotid artery; WFNS: World Federation of Neurosurgical Societies. 
for Statistical Computing, Vienna, Austria).

\section{RESULTS}

\section{Patient Demographics and Characteristics of All Ruptured Aneurysms}

Demographics and characteristics of all patients with a ruptured aneurysm are shown in Table 1. The mean age was 58.5 years, and $198(61.7 \%)$ of the total 321 patients were female. In total, 132 patients (41.1\%) with ruptured aneurysms had hypertension in their past medical history. All lab findings including lipid profile and hbAlc were within the nomal range. As to location, 122 patients (38.0\%) had ruptured aneurysms located at ACA, 90 patients (28.0\%) at MCA, and the others located in the ICA. Modified Fisher grade radiological findings were evaluated: 171 patients (53.3\%) showed grade III, and 96 patients (29.9\%) had grade IV. The average dome size of the aneurysms was $5.24 \mathrm{~mm}$, and the neck size was 3.42 $\mathrm{mm}$. According to Glasgow Coma Scores and demographics at admission, 142 patients (44.2\%) showed WFNS of grade 1 .

\section{Comparison Between MCA M-1 Trunk Aneurysm and MCA Groups}

Patient characteristics of age, sex, past medical history, and lab findings showed no definite differences between patients in the MCA M-1 trunk aneurysm group and those in the other MCA aneurysm group. Modified Fisher grade and WFNS grade also did not show differences between the two groups. However, MCA M-1 trunk aneurysms had an average dome size of $2.39 \mathrm{~mm}$ and a neck size of $2.61 \mathrm{~mm}$, while other MCA aneurysms had an average dome size of $5.79 \mathrm{~mm}$ and neck size of $3.69 \mathrm{~mm}$. In general, MCA M-1

Table 2. Univariate analysis of probable factors in the MCA group and M1 group

\begin{tabular}{|c|c|c|c|}
\hline Variables & MCA group $(n=89)$ & Ml group $(n=4)$ & $p$-value \\
\hline Age (years) & $59.56 \pm 14.19$ & $59.00 \pm 13.09$ & 0.881 \\
\hline Sex (female) & 58 (65.2\%) & $4(100 \%)$ & 0.211 \\
\hline \multicolumn{4}{|l|}{ Medical history } \\
\hline Hypertension & 32 (36.0\%) & (50.0\%) & 0.466 \\
\hline Diabetes mellitus & $1(1.1 \%)$ & 0 & 0.957 \\
\hline Ischemic heart disease & $3(3.4 \%)$ & 0 & 0.875 \\
\hline \multicolumn{4}{|l|}{ Laboratory findings } \\
\hline $\mathrm{HDL}(\mathrm{mg} / \mathrm{dL})$ & $53.46 \pm 14.02$ & $64.00 \pm 5.90$ & 0.143 \\
\hline LDL (mg/dL) & $103.48 \pm 36.58$ & $99.50 \pm 23.95$ & 0.830 \\
\hline Total cholesterol (mg/dL) & $166.19 \pm 38.08$ & $171.75 \pm 22.93$ & 0.773 \\
\hline $\mathrm{HbAlc}(\%)$ & $5.63 \pm 0.44$ & $5.80 \pm 0.32$ & 0.442 \\
\hline Systolic BP at ER (mmHg) & $162.08 \pm 34.96$ & $168.50 \pm 36.06$ & 0.720 \\
\hline Diastolic BP at ER (mmHg) & $89.28 \pm 17.99$ & $95.25 \pm 12.69$ & 0.514 \\
\hline Modified Fisher grade & & & 0.780 \\
\hline 1 & $1(1.1 \%)$ & 0 & \\
\hline$\|$ & 15 (16.9\%) & $1(25.0 \%)$ & \\
\hline III & 47 (52.8\%) & $2(50.0 \%)$ & \\
\hline IV & $26(59.2 \%)$ & 1 (25.0\%) & \\
\hline WFNS grade & & & 0.393 \\
\hline I & $36(40.4 \%)$ & $2(50.0 \%)$ & \\
\hline$\|$ & $9(10.1 \%)$ & 1 (25.0\%) & \\
\hline III & $6(6.7 \%)$ & 0 & \\
\hline IV & 21 (23.6\%) & $1(25.0 \%)$ & \\
\hline$\vee$ & 17 (19.1\%) & 0 & \\
\hline \multicolumn{4}{|l|}{ Aneurysm shape (mm) } \\
\hline Dome & $5.79 \pm 0.81$ & $2.39 \pm 0.32$ & 0.026 \\
\hline Neck & $3.69 \pm 0.51$ & $2.61 \pm 0.25$ & 0.256 \\
\hline Dome/Neck & $1.83 \pm 0.54$ & $1.01 \pm 0.21$ & 0.048 \\
\hline
\end{tabular}

MCA: middle cerebral artery; HDL: high-density lipoprotein; LDL: low-density lipoprotein; HbAlc: glycated hemoglobin; BP: blood pressure; ER: emergency room; WFNS: World Federation of Neurosurgical Societies. 
trunk aneurysms were smaller in size than other MCA aneurysms $(p=0.026)$. Also, the dome/neck ratio of MCA trunk aneurysms was lower than that of the other MCA aneurysms group $(p=0.048)$ (Table 2).

\section{Comparison Between ACA A-1 Trunk Aneurysm and ACA Groups}

Patients with ruptured ACA A-1 trunk aneurysm were younger $(p=0.001)$, with an average age of 40.78 years, than those in the ACA group, with an average age of 56.71 years. Other characteristics such as sex, past medical history, and lab findings were not significantly different between the two groups. The size of the ruptured aneurysm was smaller in the ACA A- 1 trunk aneurysm group at an average of $2.26 \mathrm{~mm}$ compared with the ACA group at an average of $4.95 \mathrm{~mm}(p=0.038)$. Like the MCA groups, the average dome/neck ratio of ACA A-1 trunk aneurysms was lower than that in the other ACA group ( $p=0.041)$ (Table 3).

\section{More About Trunk Aneurysms}

Observations of morphologic characteristics (Table 4) revealed 3 aneurysms with fusiform shape and 10 with saccular shape. The superior-posterior direction of the aneurysm dome was the most common. Associated abnormalities such as multiple aneurysms and azygous ACA were not frequent; only 1 patient had multiple aneurysms.

Perforator arteries originating from ACA A-1 and MCA M-1 trunks have a high risk for injury after surgical or interventional treatment of ruptured aneurysm. In our study, 4 patients had perforator infarct. Perforator artery injuries occurred regardless of surgical clipping or interventional coil embolization. Representative cases of patients who experienced perforator infarct just after treatment are presented below as case descriptions.

Table 3. Univariate analysis of probable factors in the ACA group and Al group

\begin{tabular}{|c|c|c|c|}
\hline Variables & ACA group $(n=112)$ & Al group $(n=9)$ & p-value \\
\hline Age (years) & $56.71 \pm 13.19$ & $40.78 \pm 15.96$ & 0.001 \\
\hline Sex (female) & 49 (43.8\%) & $6(66.7 \%)$ & 0.164 \\
\hline \multicolumn{4}{|l|}{ Medical history } \\
\hline Hypertension & 48 (42.9\%) & 3 (33.3\%) & 0.425 \\
\hline Diabetes mellitus & $8(7.1 \%)$ & 0 & 0.528 \\
\hline Ischemic heart disease & $7(6.3 \%)$ & 0 & 0.574 \\
\hline \multicolumn{4}{|l|}{ Laboratory findings } \\
\hline $\mathrm{HDL}(\mathrm{mg} / \mathrm{dL})$ & $54.63 \pm 14.23$ & $54.22 \pm 10.85$ & 0.933 \\
\hline $\mathrm{LDL}(\mathrm{mg} / \mathrm{dL})$ & $102.71 \pm 31.21$ & $90.78 \pm 39.89$ & 0.233 \\
\hline Total cholesterol (mg/dL) & $175.76 \pm 96.26$ & $156.22 \pm 38.90$ & 0.748 \\
\hline $\mathrm{HbAlc}(\%)$ & $5.72 \pm 0.59$ & $5.33 \pm 0.56$ & 0.051 \\
\hline Systolic BP at ER (mmHg) & $162.94 \pm 30.83$ & $148.89 \pm 59.32$ & 0.211 \\
\hline Diastolic BP at ER (mmHg) & $90.87 \pm 16.41$ & $87.67 \pm 16.01$ & 0.554 \\
\hline Modified Fisher grade & & & 0.856 \\
\hline | & 0 & 0 & \\
\hline$\|$ & $17(15.2 \%)$ & $1(11.1 \%)$ & \\
\hline III & $65(58.0 \%)$ & $6(66.7 \%)$ & \\
\hline IV & $30(26.8 \%)$ & $2(22.2 \%)$ & \\
\hline WFNS grade & & & 0.121 \\
\hline 1 & $50(44.6 \%)$ & $4(44.4 \%)$ & \\
\hline$\|$ & 14 (12.5\%) & $1(11.1 \%)$ & \\
\hline III & $11(9.8 \%)$ & 0 & \\
\hline IV & $15(13.4 \%)$ & $4(44.4 \%)$ & \\
\hline V & $22(19.6 \%)$ & 0 & \\
\hline \multicolumn{4}{|l|}{ Aneurysm shape $(\mathrm{mm})$} \\
\hline Dome & $4.95 \pm 0.98$ & $2.26 \pm 0.58$ & 0.038 \\
\hline Neck & $3.17 \pm 0.86$ & $2.26 \pm 0.66$ & 0.119 \\
\hline Dome/Neck & $1.61 \pm 0.65$ & $0.90 \pm 0.19$ & 0.041 \\
\hline
\end{tabular}

ACA: anterior cerebral artery; HDL: high-density lipoprotein; LDL: low-density lipoprotein; HbAlc: glycated hemoglobin; BP: blood pressure; ER: emergency room; WFNS: World Federation of Neurosurgical Societies. 
Table 4. Overview of characteristics of patients with trunk aneurysm

\begin{tabular}{|c|c|c|c|c|c|c|c|c|c|c|c|c|c|}
\hline Case no. & Sex & Age & Comorbidity & Location & Shape & Direction & Dome & Neck & m-FS* & $\mathrm{GCS}^{+}$ & Management & $\mathrm{mRS}^{\ddagger}$ & Perforator infarction ${ }^{\S}$ \\
\hline 25 & $\mathrm{~F}$ & 42 & & ACA(A1), L & Fusiform & Superior & 0.87 & 1.81 & 3 & 15 & Clip & 0 & 0 \\
\hline 65 & $\mathrm{~F}$ & 71 & HTN & $A C A(A 1), L$ & Fusiform & Superior & 2.57 & 4.88 & 3 & 3 & SACE & 6 & 0 \\
\hline 68 & $\mathrm{~F}$ & 76 & HTN & $M C A(M 1), R$ & Saccular & Inferior & 2.76 & 3.22 & 4 & 3 & Coil & 6 & 0 \\
\hline 79 & $\mathrm{~F}$ & 40 & HTN & ACA(AI), L & Fusiform & Posterior & 0.98 & 2.76 & 4 & 13 & SACE & 3 & 1 \\
\hline 100 & $\mathrm{~F}$ & 45 & & MCA(M1), L & Saccular & Inferior & 3.09 & 2.39 & 3 & 15 & Clip & 1 & 1 \\
\hline 106 & $\mathrm{~F}$ & 32 & & $A C A(A 1), L$ & Saccular & Posterior & 2.28 & 1.72 & 3 & 15 & Clip & 3 & 1 \\
\hline 111 & M & 58 & & $A C A(A 1), L$ & Lobulated & Superior & 3.21 & 3.08 & 3 & 14 & Clip & 1 & 0 \\
\hline 183 & $F$ & 22 & & ACA (A1), L & Saccular & Superior & 2.57 & 3.34 & 3 & 15 & Coil & 1 & 0 \\
\hline 198 & $\mathrm{~F}$ & 54 & & MCA(M1), L & Saccular & Posterior & 2.7 & 3 & 3 & 15 & Coil & 0 & 0 \\
\hline 221 & $\mathrm{~F}$ & 61 & HTN & $M C A(M 1), R$ & Saccular & Superior & 3.39 & 2.92 & 2 & 15 & Clip & 2 & 0 \\
\hline 264 & $\mathrm{~F}$ & 39 & & $A C A(A 1), R$ & Saccular & Posterior & 3.08 & 3.06 & 4 & 15 & Coil ${ }^{\|}$ & 2 & 1 \\
\hline 280 & M & 42 & HTN & $\mathrm{ACA}(\mathrm{A} 1), \mathrm{R}$ & Saccular & Medial & 3.22 & 2.5 & 2 & 15 & Clip & 1 & 0 \\
\hline 283 & $M$ & 21 & & $A C A(A 1), R$ & Saccular & Posterior & 2.44 & 1.81 & 3 & 15 & Clip & 1 & 0 \\
\hline
\end{tabular}

F: female; M: male; HTN: hypertension; ACA: anterior cerebral artery; L: left; MCA: middle cerebral artery; R: right; m-FS: modified Fisher scale; GCS: Glasgow Coma Scale; mRS: modified Rankin Scale; SACE: stent-assisted coil embolization.

${ }^{*}$ Accounts for thick cistern and hemorrhage to predict symptomatic vasospasm. ${ }^{+}$The state of consciousness at the time of admission. ${ }^{\ddagger} \mathrm{A}$ com-

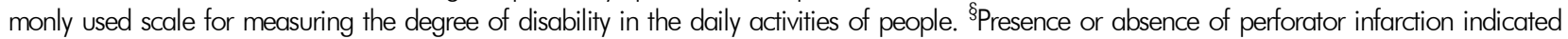
in the column. "Coil embolization without stent assist.

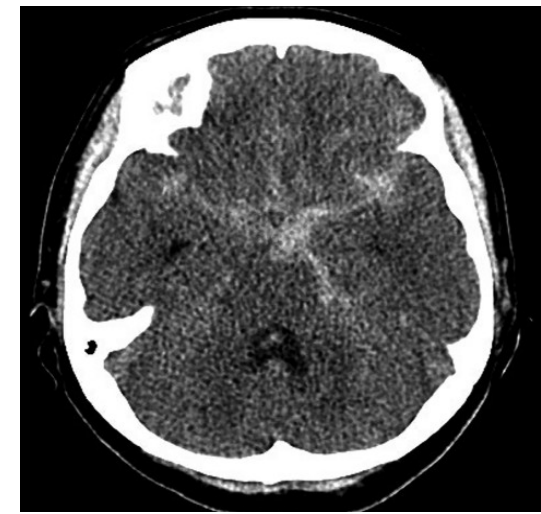

Fig. 2. Initial brain computed tomography results showing a diffuse subarachnoid hemorrhage.

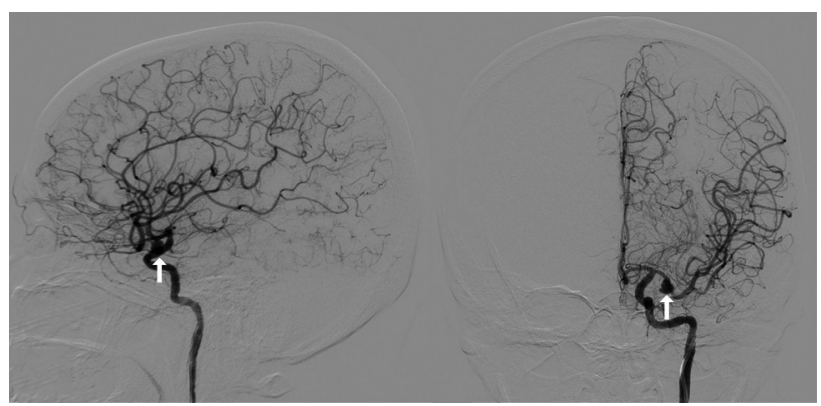

Fig. 3. Transfemoral cerebral angiography showing left middle cerebral artery M-1 trunk aneurysm.

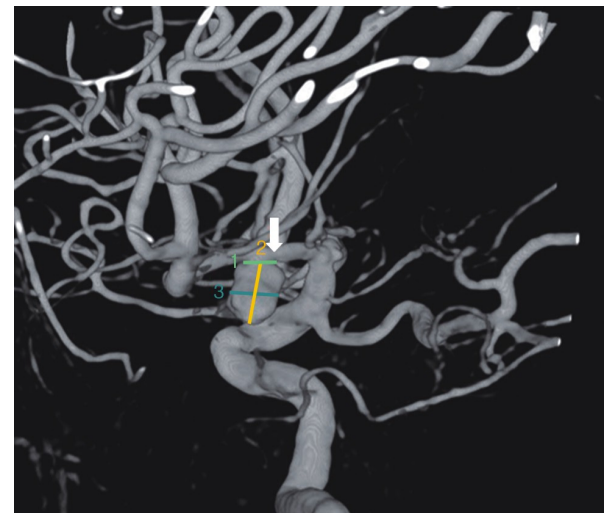

Fig. 4. The 3-dimensional reconstruction view showing a ruptured aneurysm in more detail (arrow: middle cerebral artery $\mathrm{M}-1$ segment).

\section{Case Descriptions}

\section{Case 1.}

A 45-year-old woman was admitted to our hospital due to a severe headache. Imaging studies showed SAH from a ruptured left MCA M-1 trunk aneurysm (Fig. 2-4). Surgical management with aneurysmal clipping was planned. After the operation, the patient suffered right-side weakness. Brain diffusion-weighted magnetic resonance imaging (DW-MRI) showed acute infarction on the left basal ganglia (Fig. 5), the territory where the MCA perforators supply blood flow, which was probably caused by interruption of MCA perforator arteries. 


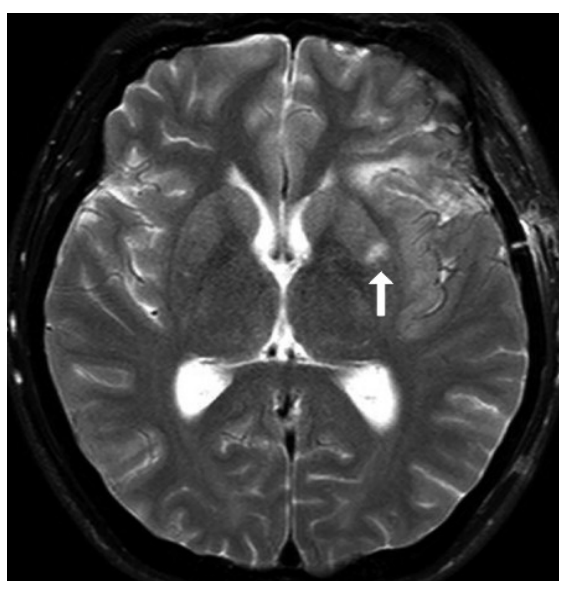

Fig. 5. Brain diffusion-weighted magnetic resonance imaging after surgical clipping (arrow: left basal ganglia acute infarction is shown).

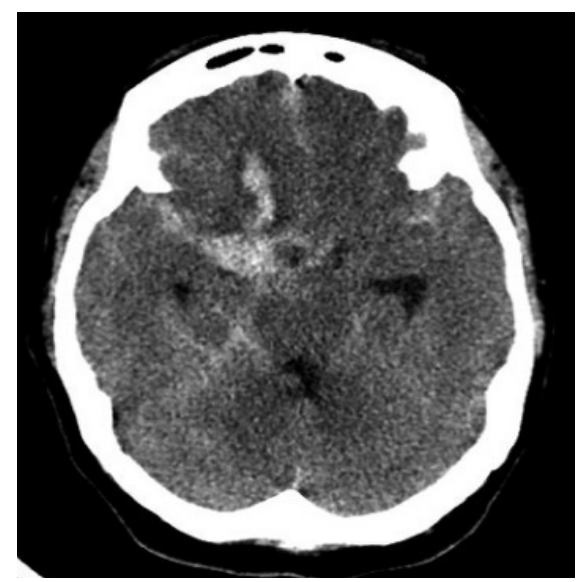

Fig. 6. Initial brain computed tomography scan showing a large amount of subarachnoid hemorrhage and intracerebral hemorrhage in the right frontal lobe base.

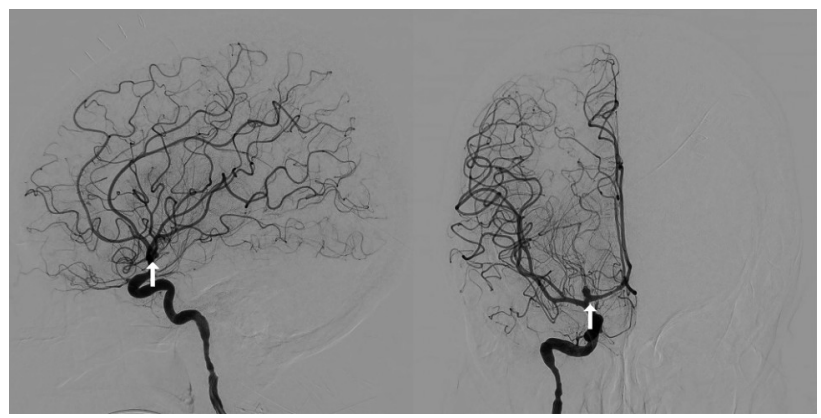

Fig. 7. Transfemoral cerebral angiography showing a right anterior cerebral artery trunk aneurysm.

\section{Case 2.}

A 39-year-old woman was admitted to our hospital due to a thunderclap headache. Imaging studies showed SAH from a ruptu-

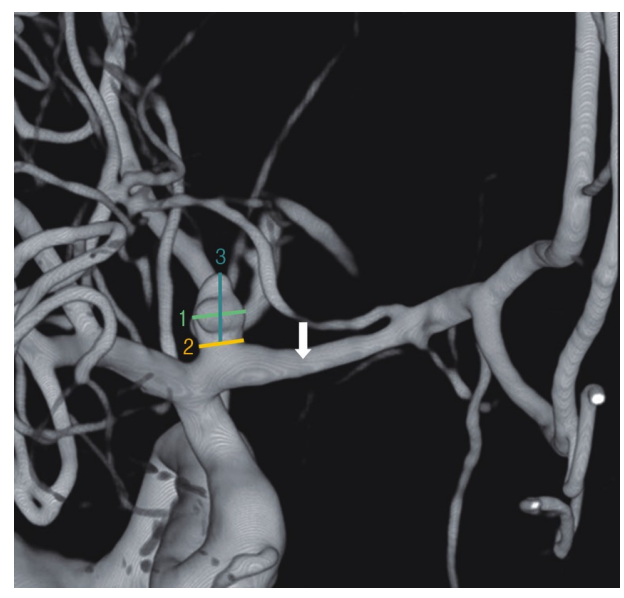

Fig. 8. The 3-dimensional reconstruction view showing a ruptured aneurysm in more detail (arrow: anterior cerebral artery A-1 segment).

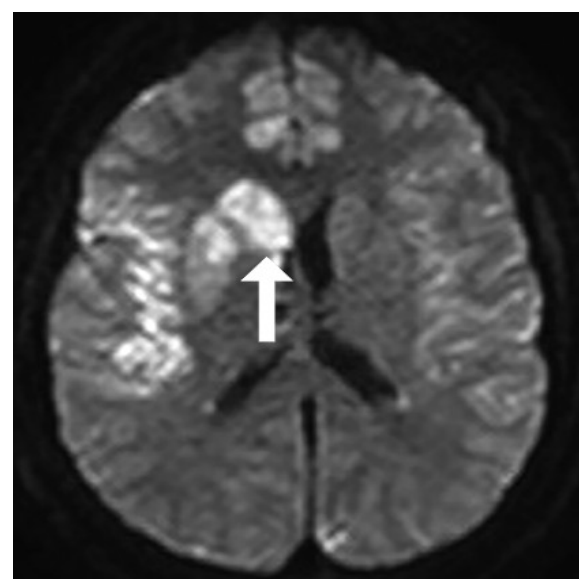

Fig. 9. Brain diffusion-weighted magnetic resonance imaging after interventional coil embolization (arrow: right basal ganglia acute infarction).

red right ACA A-1 trunk aneurysm (Fig. 6-8). Interventional management with aneurysmal coil embolization was planned. After the intervention, the patient showed left side weakness. Brain DW-MRI showed acute infarction on the right basal ganglia (Fig. 9), the region where the ACA perforators supply blood flow, which was probably caused by interruption of ACA perforator arteries.

\section{DISCUSSION}

\section{Patient Demographics and Characteristics of Trunk Aneurysms}

Ruptured trunk aneurysms are rare. Ruptured and un-ruptured ACA A- 1 aneurysms account for approximately $4 \%$ of all aneurysms $^{9,18,20)}$, and ruptured and un-ruptured MCA M-1 aneurysms account for approximately $3 \%$ of all aneurysms ${ }^{10,11)}$. In this study, 
we focused on patients with ruptured aneurysms. In total, 9 patients (2.7\% of all ruptured aneurysms) had ruptured ACA A-1 trunk aneurysms, and 4 patients (1.2\% of all ruptured aneurysms) had ruptured MCA M-1 trunk aneurysms. Thus, it is acceptable that trunk aneurysms with ruptured are rare as all trunk aneurysms including ruptured or un-ruptured.

Some studies suggest that trunk aneurysms tend to occur in those who are younger $5,6,14,18)$; our results were similar. The average age of ACA A-1 trunk aneurysm patients is 42.9 years old, which is younger than patients with other ACA aneurysms.

In our study, ruptured trunk aneurysms were smaller than other ruptured aneurysms. ACA trunk aneurysms had an average dome of $2.48 \mathrm{~mm}$ with a neck of $2.72 \mathrm{~mm}$, and MCA trunk aneurysms had an average dome of $2.98 \mathrm{~mm}$ and a neck of $2.88 \mathrm{~mm}$. The trunk aneurysms' dome/neck ratios were generally lower than those of other aneurysms, which was similar to the previous studies ${ }^{4,5,9,11,18)}$.

In our case studies, ACA A-1 trunk aneurysms tended to be directed superior and posterior, while MCA M-1 trunk aneurysms did not show direction tendency. Also, ACA A-1 trunk aneurysms tend to be located at the proximal or distal portion of ACA A-1, we assumed that those curves or branch points of ICAB to ACA A-1 portion and ACA A-1 portion to AComA are under greater hemodynamic stress.

There are several differences between trunk and other aneurysms. For example, aneurysmal size, dome/neck ratio, formation type, and patient demographics (such as age) all differ between the trunk and other aneurysms. These differences might arise from interfering factors or the pathophysiology of trunk aneurysms compared with other aneurysms.

\section{Intracranial Hemorrhagic Formation from Trunk Aneurysms}

There are many cases where $\mathrm{SAH}$ accompanies intracerebral hemorrhage $(\mathrm{ICH})$ and intraventricular hemorrhage $(\mathrm{IVH})^{8,12)}$; thus, many physicians define Fisher grade for those who suffer from $\mathrm{SAH}$ based on the presence of $\mathrm{ICH}$ and $\mathrm{IVH}$.

Fisher grade difference between trunk aneurysms and other aneurysms was not significant, perhaps due to the small number of cases. However, of the 13 patients with trunk aneurysm rupture, only 1 had ICH and 2 had IVH. We assume this was because the size of ruptured trunk aneurysms generally is smaller than that of other aneurysms. Smaller aneurysmal rupture has lower tendency for massive hematoma volume and accompanying $\mathrm{ICH}$ or $\mathrm{IVH}^{21)}$.

\section{Other Associated Abnormalities of Trunk Aneurysms}

Previous studies about trunk aneurysms suggest that the main characteristics of trunk aneurysms are association with abnormality; for example, multiple aneurysms, AComA fenestration, or ACA A-1 aplasia $^{1,6,10,11,13,14,18,20)}$. However, in this study, only 1 case with multiple aneurysms was observed, and no other abnormalities were seen. There may be several reasons for the results to be different from previous studies, such as the small number of cases. However, considering that the number of cases in previous studies was also not many, it seems that further research on this should be continued.

\section{Perforator Artery Infarctions}

Of the 13 patients with ruptured trunk aneurysms, 4 had worsened neurologic deterioration just after surgical clipping or interventional coil embolization. Perforator arteries originating from ACA A-1 or MCA M-1 trunk aneurysms are frequent ${ }^{3,7,22)}$ due to specific anatomical features, increasing the possibility of perforator artery injury compared to other aneurysms when surgical or interventional management is needed.

Recently, diagnostic tools such as computed tomography (CT) angiography and TFCA for ruptured aneurysms have been developed. These tools show accurate vascular anatomies, especially geometries of aneurysms and neighboring arterial branches in the aneurysmal neck or base. However, in many cases, small perforator arteries are not explored adequately. For example, CT angiography and TFCA did not show clear perforator arteries around the aneurysm even when such an artery was present during the attempt to clip the ruptured aneurysm. Also, unexpected perforator artery occlusion has been shown after coil embolization of a ruptured aneurysm. Therefore, it is wise to analyze ruptured aneurysms in detail using correct diagnostic tools to determine whether surgical or interventional management is better for the patients. The possibility of perforator artery injury must always be considered.

The present study has several limitations. First, it is a retrospective study design instead of a randomized controlled study, which could have allowed bias in patient selection and demographic data. Second, as we had a small number of cases all based on a singleinstitution experience, statistical analyses and results did not allow firm conclusions. Further studies with sufficient sample sizes are needed to adequately evaluate such trunk aneurysms.

\section{CONCLUSION}

Trunk aneurysms are rare and have different characteristics from other, ordinary aneurysms including smaller size, lower dome/ neck ratio, and younger age of patients. In addition, since these aneurysms are rare, more research is needed to make decisions regarding treatment. Moreover, since perforator artery injuries are highly likely due to the location of these aneurysms, smarter treatment methods should be selected based on more accurate examinations.

\section{CONFLICTS OF INTEREST}

No potential conflict of interest relevant to this article was reported.

\section{REFERENCES}

1. Bhaisora KS, Behari S, Prasadh G, Srivastava AK, Mehrotra A, Sahu RN, et al.: A I-segment aneurysms: management 
protocol based on a new classification. Neurol India 62:410416, 2014

2. Brisman JL, Song JK, Newell DW: Cerebral aneurysms. N Engl J Med 355:928-939, 2006

3. Cho YD, Lee WJ, Kim KM, Kang HS, Kim JE, Han MH: Endovascular coil embolization of middle cerebral artery aneurysms of the proximal (M1) segment. Neuroradiology 55:10971102, 2013

4. Czepko R, Libionka W, Lopatka P: Characteristics and surgery of aneurysms of the proximal (A1) segment of the anterior cerebral artery. J Neurosurg Sci 49:85-95, 2005

5. Dashti R, Hernesniemi J, Lehto H, Niemelä M, Lehecka M, Rinne J, et al.: Microneurosurgical management of proximal anterior cerebral artery aneurysms. Surg Neurol 68:366-377, 2007

6. Ding X, Nisson PL, James WS, Lawton MT, Ren S, Jia L, et al.: Aneurysms of the proximal segment of the anterior cerebral artery: A new classification system with corresponding therapeutic options. World Neurosurg 104:291-302, 2017

7. Djulejić V, Marinković S, Georgievski B, Stijak L, Aksić M, Puškaš L, et al.: Clinical significance of blood supply to the internal capsule and basal ganglia. J Clin Neurosci 25:19-26, 2016

8. Fujii M, Yan J, Rolland WB, Soejima Y, Caner B, Zhang JH: Early brain injury, an evolving frontier in subarachnoid hemorrhage research. Transl Stroke Res 4:432-446, 2013

9. Handa J, Nakasu Y, Matsuda M, Kyoshima K: Aneurysms of the proximal anterior cerebral artery. Surg Neurol 22:486490, 1984

10. Hosoda K, Fujita S, Kawaguchi T, Shose Y, Hamano S: Saccular aneurysms of the proximal (M1) segment of the middle cerebral artery. Neurosurgery 36:441-446, 1995

11. Iwama T, Yoshimura S, Kaku Y, Sakai N: Considerations in the surgical treatment of superior-wall type aneurysm at the proximal (M1) segment of the middle cerebral artery. Acta Neurochir (Wien) 146:967-972, 2004
12. Macdonald RL, Schweizer TA: Spontaneous subarachnoid haemorrhage. Lancet 389:655-666, 2017

13. Ni W, Yang H, Xu B, Xu F, Jiang H, Lei Y, et al.: Proximal middle cerebral artery aneurysms: Microsurgical management and therapeutic results. World Neurosurg 122:e907-e916, 2019

14. Park HS, Choi JH, Kang M, Huh JT: Management of aneurysms of the proximal (A1) segment of the anterior cerebral artery. J Cerebrovasc Endovasc Neurosurg 15:13-19, 2013

15. Rincon F, Rossenwasser RH, Dumont A: The epidemiology of admissions of nontraumatic subarachnoid hemorrhage in the United States. Neurosurgery 73:217-222, 2013

16. Schievink WI: Intracranial aneurysms. N Engl J Med 336:2840, 1997

17. Stoodley MA, Macdonald RL, Weir BK: Surgical treatment of middle cerebral artery aneurysms. Neurosurg Clin N Am 9:823-834, 1998

18. Suzuki M, Onuma T, Sakurai Y, Mizoi K, Ogawa A, Yoshimoto T: Aneurysms arising from the proximal (A1) segment of the anterior cerebral artery. A study of 38 cases. J Neurosurg 76:455-458, 1992

19. Thompson JW, Elwardany O, McCarthy DJ, Sheinberg DL, Alvarez CM, Nada A, et al.: In vivo cerebral aneurysm models. Neurosurg Focus 47:E20, 2019

20. Wakabayashi T, Tamaki N, Yamashita H, Saya H, Suyama T, Matsumoto S: Angiographic classification of aneurysms of the horizontal segment of the anterior cerebral artery. Surg Neurol 24:31-34, 1985

21. Wan A, Jaja BN, Schweizer TA, Macdonald RL: Clinical characteristics and outcome of aneurysmal subarachnoid hemorrhage with intracerebral hematoma. J Neurosurg 125:13441351, 2016

22. Yamamoto Y, Fukuda H, Yamada D, Kurosaki Y, Handa A, Lo B, et al.: Association of perforator infarction with clinical courses and outcomes following surgical clipping of ruptured anterior communicating artery aneurysms. World Neurosurg 107:724-731, 2017 